\title{
Existe diferença nos sintomas do Burnout entre jovens tenistas brasileiros?
}

http://dx.doi.org/10.11606/1807-5509201800040639

\author{
Hiago Luan Rolla MARTINS* \\ Franco NOCE* \\ Camila Cristina Fonseca BICALHO* \\ Felipe Gustavo dos SANTOS* \\ Daniel Alvarez PIRES** \\ Varley Teoldo da COSTA* \\ *Universidade Federal \\ de Minas Gerais, Belo \\ Horizonte, MG, Brasil. \\ **Universidade \\ Federal do Pará, \\ Belém, PA, Brasil.
}

\section{Resumo}

0 presente estudo teve c'omo objetivos a) comparar por sexo a incidência do burnout em jovens tenistas brasileiros e b) verificar se há diferenças, dentro dos grupos masculino e feminino, na percepção das dimensões do burnout nos tenistas avaliados. A amostra foi composta por 161 tenistas $(14,21 \pm 1,57$ anos), sendo 99 homens $(14,19 \pm 1,51$ anos) e 62 mulheres (14,24 $\pm 1,69$ anos). Os participantes preencheram 0 Questionário de Burnout para Atletas (QBA). Utilizou-se de estatística descritiva e inferencial não-paramétrica (Mann Whitneye Wilcoxon). Na comparação por sexo, não foram encontradas diferenças estatisticamente significativas entre homens e mulheres. Observou-se na análise intragrupo que o sexo masculino teve uma maior percepção do reduzido senso de realização em comparação com as dimensões desvalorização esportiva $(p<0,001)$ e exaustão física e emocional $(p<0,001)$. De forma semelhante, grupo do sexo feminino também apresentou uma maior percepção do reduzido senso de realização em comparação com as dimensões desvalorização esportiva $(p<0,001)$ e exaustão física e emocional $(p<0,001)$. Conclui-se que a variável sexo não influenciou na percepção do burnout para os tenistas brasileiros avaliados, sendo a dimensão reduzido senso de realização a mais percebida para os tenistas de ambos os sexos, o que sugere que estes tenistas avaliados estão insatisfeitos em relação as suas competências esportivas.

Palavras-chave: Psicologia do Esporte; Saturação Psicofisiológica; Tênis; Adolescentes.

\section{Introdução}

O Tênis de campo é uma modalidade esportiva individual de habilidades abertas que exige do atleta além do potencial físico e das habilidades técnico-táticas, as capacidades psicológicas, como equilíbrio emocional, raciocínio rápido e eficiente, e força mental/resiliência ${ }^{1,2}$. No Brasil, estima-se que mais de um milhão de pessoas pratiquem o tênis de campo, destes aproximadamente 1400 são jovens entre 12 a 18 anos e praticam o esporte em nível competitivo ${ }^{3}$. Posto isso, torna-se necessário atentar ao processo de formação dos jovens tenistas, uma vez que, em consequência da complexidade do esporte, devido às exigências físicas, psicológicas e sociais, um dos sintomas a qual estão expostos jovens tenistas é o burnout $t^{4,5}$.

No contexto esportivo, Raedeke e SMith ${ }^{6}$ se referem ao burnout como uma resposta psicofisiológica de esgotamento exibida como resultado de esforços frequentes que visam satisfazer as demandas excessivas de treinamento e competição. O burnout é fundamentado em três dimensóes: a exaustáo física e emocional, o reduzido senso de realização esportiva e a desvalorização esportiva ${ }^{6}$. A dimensão exaustão física e emocional associa-se às intensas demandas dos treinamentos e competiçóes. Já a dimensão reduzido senso de realização esportiva relaciona-se, fundamentalmente, com a insatisfação relacionada à habilidade e destreza esportiva. E, por fim, a dimensão desvalorização esportiva, associa-se a uma atitude negativa e indiferente diante do desempenho esportivo ${ }^{6}$. Em atletas, o burnout é avaliado a partir do Questionário de Burnout para Atletas-QBA, validado por Pires et al. ${ }^{7}$ e recentemente teve as suas propriedades 
psicométricas avaliadas por Guedes e Souza ${ }^{8}$ para a utilização em jovens atletas.

Atletas em formação são constantemente afetados pelo burnout ${ }^{4,5,9,10}$. De acordo Gould et al. ${ }^{4,5}$, jovens tenistas de nível competitivo são submetidos não só a demandas situacionais físicas, como cargas de treinamentos repetitivas e intensas, mas à demandas situacionais psicológicas como feedback restritivo de técnicos e familiares, proporcionando o aumento gradativo da manifestação do burnout, o que pode ocasionar o abandono prematuro da modalidade ${ }^{11,12}$. É importante considerar que, mesmo quando não há o abandono prematuro da modalidade, o burnout pode ser motivo de sofrimento psíquico para o atleta que a manifesta ${ }^{11-13}$, resultando na queda de seu desempenho ${ }^{14,15}$.

No Brasil, a incidência do burnout em jovens tenistas foi estudada por CHiminazzo e MonTAgneR $^{14}$ e de acordo com os autores $81 \%$ dos treinadores investigados já tiveram atletas que apresentaram sinais de esgotamento psicofísico característicos do burnout. CASAGRANDE et al. ${ }^{16}$ também avaliaram o burnout em tenistas da mesma faixa etária no Brasil. Segundo os resultados obtidos, tenistas com maior tempo de prática apresentaram maior percepção da exaustão física e emocional quando comparados à tenistas com menor experiência. Entretanto, ambos os estudos foram realizados a partir de uma análise geral dos tenistas, sem identificar as peculiaridades do burnout em relação ao sexo dos atletas.

Estudos internacionais, como o de WeINBERG e GoulD ${ }^{17}$ apontam relatos empíricos de casos como o da tenista Jennifer Capriati, dita como um promissor talento na modalidade que, após um processo de formação precoce mal sucedido, apresentou aos 13 anos sintomas do burnout, o que provocou uma reduçáo do seu potencial atlético de desempenho e, temporariamente, um afastamento das quadras de tênis. De Francisco et al. ${ }^{18}$ identificaram que as mulheres apresentam maiores níveis de frequência de sentimentos para a dimensão exaustáo emocional, enquanto os homens para a despersonalização. Gustafsson et al. ${ }^{19}$ afirmam que o modo como as mulheres interpretam os itens do QBA podem interferir nos valores de média geral do burnout, de modo que para as mulheres a síndrome parece ser mais agressiva. No entanto, o estudo contou com uma amostra limitada de mulheres de diferentes modalidades levantando a necessidades de novos estudos envolvendo um número maior de participantes do sexo feminino e também envolvendo modalidades específicas.

Evidências na literatura apontam que as questôes relativas ao sexo afetam diferentes constructos psicológicos em atletas, como, por exemplo, a motivação ${ }^{20}$ a emotividade e a ansiedade $e^{21}$ e habilidades psicológicas relacionadas ao coping, como lidar com adversidades e melhor desempenho sob pressão ${ }^{22}$. Além disso, estudos mostram que mulheres que praticam o esporte de rendimento podem desenvolver distúrbios hormonais que aumentam o risco de lesóes e maior exposição aos efeitos negativos do estresse, como a tríade da mulher atleta ${ }^{23-25}$. De acordo com o posicionamento oficial da American College of Sports Medicine ${ }^{26}$ a Tríade pode resultar em um declínio do desempenho físico, um aumento da morbidade clínica e psicológica e também um aumento da mortalidade. Dessa forma, no momento em que se reúnem indivíduos do sexo masculino e feminino para avaliação de determinado constructo psicológico, torna-se necessário ponderar as características e peculiaridades de cada sexo.

As questóes relativas ao tipo de sexo tornam-se ainda mais específicas quando analisados os motivos de início e abandono da prática esportiva ${ }^{27,28}$, e a influência em demandas situacionais como o apoio social para a prática esportiva e o feedback restritivo de pais e familiares, em especial, para a prática esportiva do sexo feminino em esportes com estereótipos masculinos ${ }^{29}$. Entretanto, pouco se sabe de como esta variável se manifesta em jovens tenistas brasileiros e tão pouco se existem diferenças por sexo na probabilidade de surgimento do burnout ${ }^{30}$.

Assim, com base nas evidencias apresentadas e devido ao fato de que dentro do contexto esportivo as mulheres recebem um menor apoio social dos pais e da família para a prática esportiva e feedbacks restritivos em relação à participação feminina no esporte ${ }^{29}$, o presente estudo possui a hipótese de que jovens tenistas do sexo feminino apresentam maior percepçáo do burnout quando comparado aos jovens tenistas do sexo masculino. Sendo assim, os objetivos do presente estudo foram a) comparar por sexo a incidência do burnout em jovens tenistas brasileiros e b) verificar se há diferenças, dentro dos grupos masculino e feminino, na percepção das dimensóes do burnout. 


\section{Método}

\section{Cuidados éticos}

Esse estudo foi aprovado pelo Comitê de Ética em Pesquisa da Universidade Federal de Minas Gerais sob o número CAAE - 05582312.0.0000.5149. Os indivíduos foram orientados sobre os objetivos do estudo e, posteriormente, os responsáveis pelos atletas assinaram o Termo de Consentimento Livre e Esclarecido (TCLE).

\section{Amostra}

Os participantes foram selecionados de forma não probabilística, utilizando-se o critério do voluntariado. Participaram do estudo 161 tenistas federados $(M=14,21 \pm 1,57$ anos $)$, sendo 99 homens $(M=14,19 \pm 1,51$ anos) e 62 mulheres $(\mathrm{M}=14,24 \pm 1,69$ anos). Náo foram encontradas diferença significativa $(z=-0,039 ; p=0,969)$ entre a idade dos grupos por sexo. Os participantes representam aproximadamente $10 \%$ da população de jovens tenistas segundo levantamento realizado junto à Confederação Brasileira de Tênis (CBT) a respeito da quantidade de jovens atletas federados no ano de 2012. Esses atletas se enquadram na categoria infantojuvenil, com faixa etária de 12 aos 18 anos de idade. Os atletas iniciaram a prática de tênis com média de idade de 7,86 $\pm 2,59$ anos e a média do tempo de prática como atletas federados foi de 3,71 $\pm 2,16$ anos. Foram considerados como critérios de inclusão atletas de tênis de campo, federados, inscritos em pelo menos um torneio nacional de tênis de campo, de ambos os sexos, com idade entre 12 e 18 anos.

\section{Instrumentos}

Para obtenção de informaçóes referentes à idade, tempo de prática esportiva e tempo como atleta federado foi utilizado um questionário de dados demográficos.

Como instrumento de medida para avaliação do burnout nos atletas foi utilizado o Questionário de Burnout para Atletas (QBA) ${ }^{7}$. Validado para o português por Pires, Brandão e SiLva a partir do instrumento original em inglês intitulado Athlete Burnout Questionnaire (ABQ) ${ }^{6}$. O QBA tem como objetivo mensurar as três dimensões do burnout a) exaustão física e emocional (EFE); b) a desvalorização esportiva (DES); e c) o reduzido senso de realizaçáo esportiva (RSR).

O QBA possui 15 itens, sendo cinco itens correspondentes a cada dimensão. Por exemplo, o item "Eu me sinto extremamente cansado com a minha participação no esporte" se refere à exaustão física e emocional, o item "Eu não estou alcançando muito no esporte" está relacionado ao reduzido senso de realização esportiva, enquanto que o item "Eu não estou táo interessado no esporte como eu costumava estar" é inerente à desvalorização esportiva. As respostas são dadas em uma escala do tipo Likert que varia de "Quase nunca" (1) a "Quase sempre" (5), sendo as frequências intermediárias as seguintes: "Raramente" (2), "Algumas vezes" (3) e "Frequentemente" (4). Os resultados são obtidos a partir da média aritmética das respostas dadas aos cinco itens correspondentes a cada dimensão de burnout. O valor de burnout total (BT) é calculado pela média aritmética de todos os 15 itens do instrumento. A confiabilidade do instrumento neste estudo foi $\alpha=0,745$.

\section{Procedimento}

Para a aplicação dos questionários foi realizada uma seleção, de maneira aleatória, de jovens tenistas que se encontravam em período de competição. Após esta seleção, foram realizados contatos com os treinadores com o objetivo de se obter a liberação para a participação destes indivíduos no estudo. Os dados foram coletados em dois torneios nacionais pertencentes ao calendário da CBT.

Ambos os torneios contaram com a participação de jovens tenistas de diferentes Estados do Brasil. Os atletas foram abordados antes da competição e receberam explicações a respeito dos procedimentos para se responder o QBA e como responder o questionário de dados demográficos. As coletas foram realizadas após dadas todas as instruçóes, antes dos atletas iniciarem a competição. O tempo para preenchimento do questionário e dos itens do instrumento foi de aproximadamente 10 minutos.

\section{Análise Estatística}

Para a análise de dados do burnout foi utilizada a estatística descritiva a partir da obtenção da média, mediana e desvio padrão. O teste de Alpha de 
Cronbach foi utilizado para garantir a confiabilidade do instrumento nesta amostra.

Em seguida foi utilizado o teste Kolmogorov-Smirnov para verificar a normalidade dos dados. Diante da não normalidade dos dados foi utilizado um teste de comparação de postos (teste de Mann Whitney) ${ }^{31}$

\section{Resultados}

A TABELA 1 apresenta os valores descritivos das dimensôes de burnout e burnout total dos jovens tenistas. $\mathrm{O}$ sexo feminino apresentou os maiores valores médios da dimensão reduzido senso de realização e do burnout total, enquanto o sexo masculino apresentou os maiores valores médios das dimensóes exaustão física e emocional e desvalorização esportiva. Entretanto, em ambos os sexos, os valores médios apresentados pelos tenistas brasileiros avaliados nas dimensões exaustão física e emocional e desvalorização esportiva, e no burnout total são classificados entre quase nunca e raramente, enquanto que na dimensão reduzido senso de realização esportiva foram obtidos valores médios correspondentes à frequência entre raramente e algumas vezes, o que demonstra uma baixa probabilidade de surgimento do burnout nesta amostragem. para a comparação dos escores e idades entre os sexos masculino e feminino. Já para a comparação dos escores entre as dimensóes do burnout foi utilizado o teste de Wilcoxon. Os dados foram tratados pelo software Statistical Package for the Social Sciences (SPSS) ${ }^{32}$ versão 19.0. O nível de significância adotado foi de $p<0,05$.

Observou-se que, tanto no sexo masculino quanto no feminino, a dimensão reduzido senso de realização esportiva mostrou-se a variável mais latente na percepção dos tenistas deste estudo, apresentando maiores escores. Em relação às comparações por sexo entre os tenistas, não foram encontradas diferenças significativas em nenhuma das dimensóes do burnout ou no burnout total, o que demonstra a semelhança dos estados perceptivos de ambos os sexos.

$\mathrm{Na}$ avaliação intragrupo apresentada na TABELA 2, o sexo feminino teve uma maior percepção da dimensão reduzido senso de realização esportiva quando comparado às dimensóes exaustão física e emocional e desvalorização esportiva $(p<0,001)$. A dimensão exaustão física e emocional não foi percebida como diferente da dimensão desvalorização esportiva $(p=0,31)$.

TABELA 1 - Valores descritivos das dimensões de burnout e burnout total

EFE: Exaustão física e emocional;

RSR: Reduzido senso de realização esportiva; DES: Desvalorização esportiva;

BT: Burnout total;

M: Média;

DP: Desvio padrão

EFE: Exaustão física e emocional; RSR: Reduzido senso de realização esportiva;

DES: Desvalorização esportiva. ${ }^{*} p<0,05$.

\begin{tabular}{lccccc}
\hline Dimensáo & \multicolumn{2}{c}{ Sexo feminino } & \multicolumn{3}{c}{ Sexo masculino } \\
\hline
\end{tabular}

TABELA 2 - Comparações intragrupo das percepções das dimensões do burnout (sexo feminino)

\begin{tabular}{lccc}
\hline Wilcoxon & EFE - RSR & EFE - DES & RSR - DES \\
\hline Tabela Z & $-4,51$ & $-2,16$ & $-5,63$ \\
Valor p & $*<0,001$ & 0,31 & $*<0,001$ \\
\hline
\end{tabular}

Para o sexo masculino, os resultados das comparaçóes intergrupo apresentadas na TABELA 3 também indicam uma maior percepçáo da dimensáo reduzido senso de realizaçáo esportiva, quando comparada com as dimensões exaustão física e emocional e desvalorização esportiva $(p<0,001)$. A dimensão exaustão física e emocional náo foi percebida como diferente da dimensão desvalorização esportiva $(p=0,27)$. 
TABELA 3 - Comparações intragrupo das percepções das dimensões do burnout (sexo masculino)

\begin{tabular}{lccc}
\hline Wilcoxon & EFE - RSR & EFE - DES & RSR - DES \\
\hline Tabela Z & $-3,86$ & $-2,22$ & $-6,31$ \\
Valor $\mathrm{p}$ & $*<0,001$ & 0,27 & $*<0,001$ \\
\hline
\end{tabular}

EFE: Exaustão física e emocional; RSR: Reduzido senso de realização esportiva; DES: Desvalorização esportiva. * $p<0,05$

\section{Discussão}

O presente estudo teve como objetivos a) comparar por sexo a incidência do burnout em jovens tenistas brasileiros e b) verificar se há diferenças, dentro dos grupos masculino e feminino, na percepção das dimensões do burnout.

Apesar das características e peculiaridades de cada sexo, quando comparados os resultados da percepção do burnout e suas dimensóes entre os grupos masculino e feminino de jovens tenistas brasileiros, não foram encontradas diferenças significativas. Os jovens tenistas, de ambos os sexos, apresentaram valores moderadamente reduzidos tanto para o burnout total quanto para as suas dimensões, fatores indicativos de que os mesmos não apresentavam manifestaçóes significativas do burnout. Os resultados obtidos no presente estudo corroboram o estudo de Gustafsson et al. ${ }^{19}$, o qual indicou que a maioria dos 980 atletas adolescentes suecos analisados, que pertenciam a 58 modalidades esportivas individuais e coletivas, apresentaram níveis baixos a moderados do burnout. A reduzida prevalência de burnout (entre 1 e $10 \%$ do universo de atletas) tem sido registrada em outros estudos com atletas ${ }^{33}$ Entretanto, em virtude das sérias consequências que essa síndrome provoca, afetando tanto o atleta quanto o seu entorno e impactando negativamente no seu desempenho, torna-se imperativa uma avaliação aprofundada do burnout com o intuito de reduzir ao máximo sua incidência, evitando assim eventuais desistências precoces e promovendo o bem estar psicológico do jovem atleta, fazendo com que o mesmo perceba o contexto esportivo como fonte de satisfação e realização.

No que concerne à comparaçáo por sexo, os resultados encontrados convergem com o estudo realizado por VIEIRA et al. ${ }^{34}$, no qual a variável sexo não apresentou diferença significativa quanto a percepção do burnout para os sexos feminino e masculino em 93 atletas, das categorias sub-21 e open do circuito brasileiro de vôlei de praia. Todavia, os resultados encontrados divergem da perspectiva de que o burnout apresentaria diferenças quanto à percepção entre os grupos masculino e feminino de jovens tenistas, visto que as mulheres, quando comparadas aos homens, apresentam menor apoio social para prática de esportes com estereótipos masculinos e um maior feedback restritivo de pais e familiares ${ }^{29}$.

Em um estudo com jovens tenistas, Gould et al. ${ }^{4,5}$ identificaram que demandas situacionais como o pouco apoio social para prática esportiva e o feedback restritivo de pais e familiares contribuíam para queda no desempenho dos jovens tenistas e, consequentemente, para a maior percepção da dimensão reduzido senso de realizaçáo esportiva. De fato, o grupo feminino apresentou maiores escores da dimensão reduzido senso de realizaçáo, assim como maiores escores para o burnout total. Entretanto, os valores obtidos não foram suficientes para evidenciar diferença significativa quando comparado aos valores apresentados pelo grupo masculino.

Segundo Melo, Giavoni e TrócColi ${ }^{35}$, o pouco apoio ao sexo feminino para o esporte é consequência da percepçáo de um processo cultural em que a figura feminina, na maioria das vezes, não recebe o mesmo tipo de encorajamento que a figura masculina devido aos estereótipos estabelecidos pela desejabilidade social. Por conseguinte, a figura masculina é estimulada a assumir uma postura socialmente independente, bem como a ser fisicamente ativa em atividades e em jogos esportivos, dado que, culturalmente, a figura feminina é tratada com mais suavidade e proteçáo que a figura masculina, sendo acompanhada com maior proximidade e, quando a ela é permitido participar de outras atividades, como práticas esportivas, esse fato vem condicionado a várias restriçôes.

$\mathrm{Na}$ avaliação intragrupo, a dimensão reduzido senso de realizaçáo apresentou maior escore entre todas as dimensôes em ambos os sexos, variando de raramente a algumas vezes, o que pode ser um indicativo de que os jovens tenistas não estáo plenamente satisfeitos em relação às suas habilidades, destrezas e realizações no esporte $^{6}$. Fato este comum em atletas que estáo em um período de formação e que ainda não atingiram $\mathrm{o}$ ápice de sua carreira profissional ${ }^{5}$. 
A falta de progressos no desempenho atlético e percepçóes de falta de sucesso e falta de talento são sintomas característicos da dimensão reduzido senso de realização ${ }^{4,5}$. Em um estudo com jovens tenistas, Dias e TeIXerA ${ }^{36}$ identificaram correlação entre o fato de não estar alcançando as metas traçadas e o pensamento de abandono esportivo.

Tendo em vista as dificuldades que enfrentam muitos atletas jovens devido aos valores de investimento do país no esporte, principalmente em modalidades individuais como o tênis, a maior percepção da dimensão reduzido senso de realização pode ser explicada pelo fato de que muitos atletas jovens não têm a oportunidade de alcançar o nível profissional no Brasil.

A exaustão física e emocional está associada às intensas demandas dos treinos e competiçóes ${ }^{6}$. Dessa forma, os valores reduzidos encontrados para a dimensão exaustão física e emocional nos tenistas investigados pode estar relacionado ao tempo de participação em competiçóes dos atletas, tendo em vista que tenistas investigados possuem reduzida média de tempo como atletas federados e, por consequente, não participam de um número elevado de competiçóes nesta faixa etária.

Entre as dimensões apresentadas, a desvalorização esportiva apresentou menor escore médio entre os jovens tenistas. Os sentimentos relativos à dimensão desvalorização esportiva proporcionam ao atleta um comportamento impessoal e insensível, demonstrando pouca ou nenhuma preocupação com seu rendimento e com o seu envolvimento dentro do meio esportivo ${ }^{37}$. Visto que tal dimensão se refere à falta de desejo, à despreocupaçáo e à falta de interesse em relação ao esporte ${ }^{6}$, o resultado apresentado é um indicativo de que o grupo investigado demonstra compromisso e envolvimento em relação à modalidade esportiva.

Por fim, o presente estudo apresenta algumas limitaçóes relacionadas ao delineamento transversal, o que impossibilita inferências mais precisas a respeito da percepção do burnout na amostragem de jovens tenistas brasileiros. Em virtude do burnout estar localizado no extremo de um contínuo que se inicia com reações negativas de adaptaçáo ao estresse denominadas de esgotamento e pode ter como resultado o dropout ${ }^{38}$, há uma limitação em se encontrar atletas em atividade com escores acentuados de burnout, pois existe uma grande possibilidade dos mesmos náo estarem competindo ou já terem encerrado suas carreiras atléticas, visto que o abandono esportivo constitui-se em uma das principais consequências do burnout ${ }^{12}$. Como o presente estudo foi desenvolvido com tenistas que estáo em atividade, provavelmente os mesmos não se encontrariam com escores elevados do burnout. Dessa forma, estudos longitudinais como o desenvolvido por Pires et al..$^{39}$ no âmbito do voleibol devem ser considerados em investigaçóes futuras sobre o burnout em jovens tenistas brasileiros, considerando as características e peculiaridades de casa sexo. Os estudos envolvendo as diferenças por sexo e o burnout também necessitam avançar em outras modalidades esportivas, em diferentes categorias no processo de formação de base e na categoria principal, uma vez que pouco se sabe de como estes sintomas se manifestam em um determinado esporte por um longo período de tempo e por sexo.

No âmbito das implicações práticas, os resultados do presente estudo, ainda que apontem para prevalência reduzida das dimensóes de burnout e pela similaridade dessa prevalência entre mulheres e homens, remetem à necessidade de programas de intervenção que levem em consideração os fatores pessoais e ambientais, visando à prevenção da síndrome em jovens atletas. Nesse sentido, os programas de preparação psicológica podem fazer uso de abordagens como a Terapia Cognitivo Comportamental e o mindfulness. Ambas apresentaram efeitos protetores frente à manifestaçáo do burnout no contexto esportivo ${ }^{15}$.

Conclui-se, portanto, que a variável sexo não influenciou na percepção do burnout e suas dimensóes nos jovens tenistas brasileiros avaliados. Nota-se também que os jovens tenistas brasileiros em atividade têm uma probabilidade baixa de manifestar a síndrome do burnout. Apesar disto, o reduzido senso de realização esportiva, que está associado ao fato de terem ou não sucesso na modalidade escolhida para a prática, foi a dimensão de burnout mais percebida.

\section{Abstract}

Is there difference in symptoms of Burnout between young brazilian tennis players?

This study aimed to a) compare the incidence by sex of burnout in young Brazilian tennis players, and b) check for differences within the male and female groups, in the perception of burnout dimensions in the assessed 
tennis players. The sample consisted of 161 tennis players (M14.21 \pm 1.57 years), 99 men (14.19 \pm 1.51 years) and 62 women (14.24 \pm 1.69). Participants completed the Burnout Questionnaire for Athletes (QBA). It was used descriptive and inferential statistics nonparametric (Mann Whitney and Wilconxon). Compared by gender, there was no statistically significant differences between men and women. It was observed in intra-group analysis that the males had a greater perception of the reduced sense of accomplishment in comparison to the dimensions sports devaluation $(p<0.001)$ and physical and emotional exhaustion $(p<0.001)$. Similarly, female group also had a greater perception of the reduced sense of accomplishment in comparison to the dimensions sports devaluation $(p<0.001)$ and physical and emotional exhaustion $(p<0.001)$. It can be conclude that sex did not influence the perception of burnout for Brazilian tennis players evaluated and reduced sense of accomplishment was the most perceived dimension to tennis players of both sexes, suggesting that these tennis players are unsatisfied regarding their sports skills.

KeYwords: Sport Psychology; Psychophysiological Saturation; Tennis; Adolescents.

\section{Referências}

1. Nicholls AR, Polman RCJ, Levy AR, Backhouse SH. Mental toughness in sport: achievement level, gender, age, experience, and sport type differences. Pers Individ Dif. 2009;47(1):73-5.

2. Noce F, Ferreira TS, Moreira CZ, Andrade AGP, Mello MT, Costa VT. Influência do tempo de reação simples na seleção de jovens talentos no tênis. Rev Educ Fís UEM. 2012;23(3):369-77.

3. Silva CL, Costa IP, Silva MM, Caregnato AF, Cavichiolli FR. A configuração do tênis de campo infantojuvenil brasileiro: primeiras descriçóes. Pensar en Movimiento. 2017:15(1):19-36.

4. Gould D, Tuffey S, Udry E, Loehr J. Burnout in competitive junior tennis players: a quantitative psychological assessment. Sport Psychol. 1996;10(4):322-40.

5. Gould D, Tuffey S, Udry E, Loehr J. Burnout in competitive junior tennis players: qualitative analysis. Sport Psychol. 1996;10(4):341-66.

6. Raedeke T, Smith A. Development and preliminary validation of an athlete burnout measure. J Sport Exerc Psychol. 2001;23(4):281-306.

7. Pires DA, Brandáo MRF, Silva CB. Validaçáo do questionário de burnout para atletas. Rev Educ Fis UEM. 2006;17(1):27-36.

8. Guedes DP, Sousa RO. Propriedades psicométricas do Athlete Burnout Questionnaire para uso em atletas jovens Brasileiros. Rev Educ Fís UEM 2016; 27(1):1-13.

9. Appleton PR, Hall HK, Hill AP. Relations between multidimensional perfectionism and burnout in junior-elite male athletes. Psychol Sport Exerc. 2009;10(4):457-65.

10. Smith AL, Gustafsson H, Hassmén P. Peer motivational climate and burnout perceptions of adolescent athletes. Psychol Sport Exerc. 2010;11(6):453-60.

11. Smith RE. Toward a cognitive-affective model of athletic burnout. J Sport Exerc Psychol. 1986;8(1):36-50.

12. Garcés De Los Fayos Ruiz EJ, Vallerino VT. Burnout em Iberoamerica: lineas de investigación. Cuad Psicol Deporte. 2010;10(1):47-55.

13. Raedeke T. Is athlete burnout more than just stress? A sport commitment perspective. J Sport Exerc Psychol. 1997;19(4):396-417.

14. Chiminazzo JGC, Montagner PC. Síndrome de burnout e esporte: a visão dos técnicos de tênis de campo. Rev Educ Fis UEM. 2009;20(2):217-23.

15. Gustafsson H, DeFreese JD, Madigan DJ. Athlete burnout: review and recommendations. Curr Opin Psychol. 2017;16:109-13.

16. Casagrande PO, Andrade A, Viana MS, Vasconcellos DIC. Burnout em tenistas brasileiros infanto-juvenis. Motri. 2014;10(2):60-71.

17. Weinberg RS, Gould D. Fundamentos da psicologia do esporte e do exercício. 4a ed. Artmed: Porto Alegre; 2008.

18. De Francisco C, Garcés De Los Fayos Ruiz EJ, Arce C. Burnout en deportistas: prevalencia del síndrome a través de dos medidas. CPD. 2014;14(1):29-38.

19. Gustafsson H, Kenttä G, Hassmén P, Lundqvist C. Prevalence of burnout in competitive adolescent athletes. Sport Psychol. 2007;21:21-37. 
20. Li C, Wang CJ, Kee YH. Burnout and its relations with basic psychological needs and motivation among athletes: a systematic review and meta-analysis. Psychol Sport Exerc. 2013;14(5):692-700.

21. Craft L, Magyar M, Becker B, Feltz D. The relationship between the competitive state anxiety inventory-2 and sport performance: a meta-analysis. J Sport Exerc Psychol. 2003;25(1):44-65.

22. Coimbra DR, Bara Filho M, Andrade A, Miranda R. Habilidades psicológicas de coping em atletas brasileiros. Motri. 2013;9(1):95-106.

23. Pardini DP. Alteraçóes hormonais da mulher atleta. Arq Bras Endocrinol Metab. 2001;45(4):343-51.

24. Perini TA, Oliveira GL, Dantas PMS, Fernandes PR, Fernandes Filho J. Investigação dos componentes da tríade da mulher atleta em ginastas. Rev Educ Fís UEM. 2009;20(2):225-33.

25. DiFiori JP, Benjamin HJ, Brenner JR, Gregory A, Jayanthi N, Landry GL, Luke A. Overuse injuries and burnout in youth sports: a position statement from the American Medical Society for Sports Medicine. Br J Sports Med. 2014;48:287-8.

26. Nattiv A, Loucks AB, Manore MM, et al. American College of Sports Medicine Position Stand: The female athlete triad. Med Sci Sports Exerc. 2007;39(10):1867-82.

27. Carmo J, Matos F, Ribas P, Miranda R, Bara Filho M. Motivos de início e abandono da prática esportiva em atletas brasileiros. HURevista. 2009;35(4):257-64.

28. Bara Filho MG, Guillén Garcia F. Motivos do abandono no esporte competitivo: um estudo retrospectivo. Rev Bras Educ Fís Esporte. 2008;22(4):293-300.

29. Cardoso BLC, Assumpção LOT, Melo GF, Silva JVP, Sampaio TMV. Mulheres no boxe: Percepçóes sociais de uma luta sem luvas e fora dos ringues. Motri. 2012;8(2):259-68.

30. Goodger K, Gorely T, Lavallee D, Harwood C. Burnout in sport: a systematic review. Sport Psychol. 2007;21(2):127-51.

31. Pasquali L. Instrumentação psicológica: fundamentos e práticas. Porto Alegre: Artmed; 2010.

32. Dancey CP, Reidy J. Estatística sem matemática para psicologia: usando SPSS para Windows. Porto Alegre: Artmed; 2006.

33. Gustafsson H, Kenttä G, Hassmén P. Athlete burnout: an integrated model and future research directions. Int Rev Sport Exerc Psychol. 2011;4(1):3-24.

34. Vieira LV, Carruzo NM, Aizava PVS, Rigoni PAG. Análise da síndrome de "burnout" e das estratégias de "coping" em atletas brasileiros de vôlei de praia. Rev Bras Educ Fís Esporte. 2013;27(2):269-76.

35. Melo GF, Giavoni A, Tróccoli BT. Estereótipos de gênero aplicados a mulheres atletas. Psic Teor Pesq. 2004;20(3):251-6.

36. Dias MH, Teixeira MA. Estudo exploratório sobre o abandono do esporte em jovens tenistas. Rev Bras Psicol Esporte. 2007;1(1):1-18.

37. Curran T, Appleton PR, Hill AP, Hall HK. The mediating role of psychological need satisfaction in relationships between types of passion for sport and athlete burnout. J Sports Sci. 2013;31(6):597-606.

38. Raedeke T, Lunney K, Venables K. Understanding athlete burnout: coach perspectives. J Sport Behav. 2002;25(2):181-206.

39. Pires DA, Debien PB, Coimbra DR, Ugrinowitsch H. Burnout e coping em atletas de voleibol: uma análise longitudinal. Rev Bras Med Esporte. 2016; 22(4):277-81

ENDEREÇO

Varley Teoldo da Costa

Universidade Federal de Minas Gerais

Av. Presidente Carlos Luz, 4664,

Campus - Pampulha - 31270-901

Belo Horizonte - MG - BRASIL

e-mail: vtcosta@hotmail.com
Recebido para publicação: 28/07/2015

Revisão: 20/09/2017

Aceito: 29/12/2017 